\title{
Agricultura de montaña en el País Vasco. Balance de una década
}

\author{
Juan Cruz Alberdi Collantes*
}

\begin{abstract}
RESUMEN
Las politicas de desarrollo rural impulsadas desde la CE tienen escasa incidencia en el espacio vasco-atlántico debido, en gran medida, a que los esfuerzos presupuestarios de las distintas instituciones están dirigidos a promover actividades en el medio urbano. Aun asi, en este territorio funcionan unas entidades de desarrollo rural desde hace más de una década, las Asociaciones de Agricultura de Montaña. En este artículo describimos el funcionamiento de estos entes de desarrollo rural, centrándonos en el modelo guipuzcoano, que dirige sus actuaciones principalmente al desarrollo agrícola y que, ante la faita de iniciativa local, se constituye en promotor $y$ ejecutor de sus propios programas.
\end{abstract}

PALABRAS CLAVE: caserío, Gipuzkoa, País Vasco, agricultura, desarrollo rural, Agricultura de Montaña.

\section{ABSTRACT}

The Europe Rural development policy in the Atlantic Basque Country has not an important present. Europe development programs are destining to promote industrial and urban project. However, in this country there are some institutions to promoting rural development actuation, the mountain farm associations. In this paper we observed their composition around the studying of the model of farm development in Gipuzkoa. In this area, the farm mountains associations are promoting specially farm projects and are doing the role of promotion and ejection of themselves rural programs because there isn't any local initiative.

KEY WORDS:

Gipuzkoa, Basque Country, agriculture, rural development, Mountain farm.

* Departamento de Geografía de la UPV. 
Con la aprobación del decreto del Gobierno Vasco, 394/1985 y la consecuente creación de las Asociaciones de Agricultura de Montaña se inicia en el País Vasco una política de desarrollo de su medio rural, que se va concretando en diferentes intervenciones sin apenas interrupciones hasta la actualidad.

En principio, el $80 \%$ del territorio del País vasco es declarado como zona de Agricultura de Montaña y dentro de éste el $100 \%$ del territorio Guipuzcoano. A partir de 1986 se forma la primera Asociación en Gipuzkoa, en la comarca de Urola-Costa, proceso que concluye siete años después, en 1993, con la creación de la Asociación de Donostialdea-Bidasoa.

Tras una década de funcionamiento, estas Asociaciones mantienen sus objetivos y estructuras iniciales, apenas alteradas por otra serie de planes de desarrollo rural que sí han tenido incidencia en otros territorios vascos. El carácter urbano de todos los valles de la provincia de Gipuzkoa impide que sus zonas rurales se beneficien de nuevos programas de desarrollo rural, a la par que aporta un progreso peculiar al de Agricultura de Montaña en estas áreas.

En esta investigación recogemos algunas de las particularidades del funcionamiento de estas Asociaciones, analizamos las actividades que han promocionado y realizamos una crítica de su trayectoria, fundamentándonos en los proyectos que éstas han iniciado desde inicios de la década de los noventa.

\section{BREVE INTRODUCCIÓN A LAS FIGURAS DE DESARROLLO RURAL EN EL PAIIS VASCO}

El sector agrícola del Pais Vasco está sumido en una crisis estructural que está trayendo consigo una reducción progresiva del número de agricultores. Las zonas rurales soportan una pérdida continua de población y la que continúa residiendo lo hace a cuenta de mantener unas diferencias económicas y sociales comparativas importantes con las del medio urbano.

Es a partir de la designación por parte de la Comisión Europea del programa de Agricultura de Montaña cuando el Gobierno Vasco decide crear las Asociaciones de Agricultura de Montaña. El desarrollo del medio rural, la mejora de sus condiciones de vida, de su situación social, la incorporación de servicios etc., se convierten en la máxima preocupación de estos entes y su labor es acompañada con el apoyo presupuestario de las instituciones.

Se inician toda una serie de programas dirigidos a impulsar el desarrollo del medio rural, que, marcados por las líneas de actuaciones generales 
de la Política Agraria Comunitaria, presentan en la zona atlántica del País Vasco unas particularidades características.

La consideración por los fondos de cohesión europeos de toda el área atlántica del País Vasco, incluidas sus zonas rurales, como Objetivo 2, es decir, como zona en declive industrial, según el reglamento (CE) n. ${ }^{\circ}$ $1260 / 1999^{1}$, es la que le condiciona su evolución. Únicamente una serie de distritos de las tres capitales del País Vasco han perdido la consideración de Objetivo regional $n .{ }^{\circ} 2$ y han pasado a ser zonas de ayuda transitoria debido a exigencias de la población asistida.

La evolución de los distintos programas de desarrollo rural que se han puesto en marcha es, incluso anterior. Coincidiendo con la situación habitual del medio rural español, hasta la aprobación de la ley de Agricultura de Montaña en 1982, la política de desarrollo rural en el País Vasco consistía en mejoras en el sector agrario. La incorporación a la UE en 1986 significó la consolidación de los nuevos enfoques de desarrollo rural, avanzando hacia un enfoque integrado, endógeno y participativo?

Entre los distintos programas potenciados ha sido el de Agricultura de Montaña, iniciado en 1986, el que ha aportado al desarrollo de las áreas rurales de la zona atlántica vasca un carácter unitario. Por cada comarca surge una Asociación de Agricultura de Montaña, englobada por las entidades locales y las Asociaciones y Sindicatos del sector que ejercen su actividad en la zona. Surgen con el objeto de mejorar las condiciones y la calidad de vida de las áreas del medio rural y para potenciar diversas iniciativas de desarrollo endógeno: cooperativas, turismo rural, infraestructuras, elaboración y venta de productos, artesanado, etc.

En 1991, la Comisión Europea crea la iniciativa comunitaria LEADER I, con el propósito de impulsar una línea integradora, intención que se subraya en los objetivos, a la vez que se hace explícito su enfoque endógeno y local. Manifestaba también su vocación demostrativa, que sirviera de ejemplo para animar a intervenciones privadas en otras áreas a la vez que de experiencia para posteriores intervenciones institucionales. Estos programas actúan a través de subvenciones aplicándose a determinadas

\footnotetext{
1 Reglamento CE n. $1260 / 1999$ de 21 de Junio de 1999 por el que se establecen disposiciones generales de los Fondos Estructurales.

2 «De este modo, tan sólo en una década, la concepción de desarrollo rural ha cambiado completamente desde una clara perspectiva top-dow a un enfoque integrado, endógeno y participativo. Sin embargo, en la puesta en práctica de este enfoque existen problemas para asumir los principios y requerimientos de dicho enfoque y por el momento sólo pueden destacarse algunas mejoras" (Esparcia, J. y Nogueras, J., 1999, pág. 82).
} 
áreas incluidas entre los objetivos 1 y $5 \mathrm{~b}$, presentes estos últimos en el País Vasco (Corbera, 1999).

Las zonas Objetivo $5 b$, que no alcanzan a acaparar el $20 \%$ del total del territorio atlántico vasco, corresponden mayormente a comarcas alejadas de los núcleos urbanos e industriales o a zonas de Montaña en las que la actividad agrícola es casi su única actividad económica. La emigración ha sido patente en las últimas cinco décadas y la población envejecida es mayoritaria. En éstas, a través de los fondos ligados al $5 \mathrm{~b}$, se impulsan todo tipo de proyectos, como el turismo rural, formación, apoyo técnico, etc. Pero quizá lo más interesante son los proyectos incluidos en la medida destinada a potenciar el desarrollo industrial, la que más inversión ha absorbido, normalmente pabellones industriales con actividades destinadas a diversificar la actividad productiva de la comarca (Gobierno Vasco, 1996).

El resto de áreas rurales, a pesar de presentar unas condiciones similares (emigración, población envejecida, actividad agrícola como única actividad, etc...), no fueron consideradas zona $5 b$ y se englobaron dentro del Objetivo 2, como áreas industriales en declive. Este territorio, la mayoría del espacio ordenado por el caserío, se encuentra, si cabe, en una situación todavía más difícil respecto a su medio. El abandono masivo de la actividad agrícola, con una población atraída por el empleo urbano, se va materializando progresivamente. La vida del municipio se va perdiendo, dando paso a usos residenciales, en barrios dormitorio, mientras su patrimonio y su medio se va degradando.

Estas zonas se ven necesitadas de actuaciones en materia de desarrollo rural, preferentemente agrícola, que permita fijar población y mantener el uso agrario del medio ${ }^{3}$. La declaración de zona en declive industrial, si bien no está cerrada a proyectos de desarrollo de estos núcleos, desvía la mayor parte de sus fondos al medio urbano: formación, promoción de nuevas zonas industriales, infraestructuras, etc. El espacio rural atlántico, de este modo, queda mayoritariamente fuera de los fondos de desarrollo rural.

3 La peculiaridad que parece presentar el caserío vasco dentro del proceso de reestructuración observado estriba en la intensidad con la que se está materializando el abandono de la función agraria, muy superior a la producida en otras áreas o regiones europeas. AINZ llega a cuantificarlo y apunta que tan sólo el $5 \%$ de los caseríos se caracteriza por constituir explotaciones dinámicas y modernizadas en las que la actividad principal es la agraria. En el resto, la labor es residual y supone por lo general un escaso complemento económico a las rentas familiares. De sus afirmaciones también se desprende que la estrategia de la complementariedad de la renta agraria en la propia explotación apenas tiene importancia y tan sólo alrededor del $2 \%$ obtendrian aportaciones económicas a partir de ésta (1999, pág. 332). 
Con la puesta en marcha de la Agenda 2000, los esfuerzos políticos han ido dirigidos a ampliar la zona de afección que anteriormente comprendía el $5 \mathrm{~b}$. Se delimita un territorio considerado con mayor prioridad para la aplicación de la iniciativa comunitaria LEADER + ${ }^{4}$. Esta zona congrega a un total de municipios, lo que supone el $54,7 \%$ de la extensión de la Comunidad Autónoma y cuya homogeneidad viene caracterizada no sólo por sus condiciones orográficas, sino también por los aspectos socioeconómicos de una población que habita en una zona de Agricultura de Montaña y que se corresponden con una baja densidad poblacional $(18,7$ $\left.\mathrm{hab} / \mathrm{km}^{2}\right)$.

A pesar de la extensión que pretende incluir el programa LEADER + muchas áreas, preferentemente de Gipuzkoa, no se van a ver beneficiadas por los fondos de desarrollo rural que en él se recogen. La propia estructura física de Gipuzkoa, ordenada en torno a estrechos corredores en los que se entremezclan actividades urbanas y agrarias, impide la consideración de sus zonas rurales como marginales y ello a pesar de que, una vez superado el fondo del valle, sean los usos agrarios los prioritarios, a los que se les destina más del $80 \%$ de la superficie de estas comarcas ${ }^{5}$.

El carácter montañoso del País, sin embargo, si justifica la pervivencia de los programas de Agricultura de Montaña, extendidos a toda la superficie del territorio guipuzcoano y es este propósito el que estructura las acciones de desarrollo rural en este territorio. La incidencia del resto de iniciativas es escasa y localizada.

\section{REGULACIÓN DEL RÉGIMEN ESPECÍFICO DE AGRICULTURA DE MONTAÑA}

Las Asociaciones de Agricultura de Montaña se constituyen a partir de la entrada en vigor del DECRETO 394/1985, sobre el Régimen específico de la Agricultura de Montaña (AM) en la Comunidad Autónoma del País Vasco.

Los objetivos que se persigue alcanzar en las zonas de AM se recogen en el art. 1:

4 Comunicación de la comisión de los estados miembros de 2000 por la que se fijan orientaciones sobre la iniciativa comunitaria de desarrolio rural LEADER +.

5 El Censo Agrario de 1999 clasifica como agrarias en la comarca más urbanizada de Gipuzkoa, la de Donostialdea-Bidasoa, un total de $28.817 \mathrm{Ha}$, el $76,5 \%$ de su superficie geográfica, trente al $81,2 \%$ que registra el conjunto de la provincia. 
a) Asegurar a las explotaciones agrarias de las Zonas de Agricultura de Montaña unos ingresos mínimos.

b) Situar a las Zonas de Agricultura de Montaña a unos niveles mínimos de equipamientos sociales e infraestructuras.

c) Asegurar una combinación de rentas de forma que ingresos industriales, turísticos, artesanales y agrarios puedan complementarse entre sí.

d) Respetar el medio natural y realizar e impulsar acciones que tiendan a su conservación.

e) Impulsar acciones destinadas a preservar el patrimonio históricocultural.

Para que una comarca sea catalogada como Zona de Agricultura de Montaña deberá cumplir una serie de condiciones que son recogidas en el art. 2:

a) Tener una pendiente media superior al $20 \%$ o una diferencia entre las cotas extremas de una superficie agraria superior a 400 metros.

b) Tener vocación predominantemente agraria y concurrir en ellas simultáneamente circunstancias de altitud y pendiente que, sin llegar a los valores indicados en el párrafo anterior, den lugar a circunstancias excepcionales limitativas de la producción agraria.

En base a ello se establecen las siguientes Comarcas de AM (art. 3):

$\begin{array}{lll}\text { ÁLAVA } & \text { BIZKAIA } & \text { GIPUZKOA } \\ \text { Valles alaveses } & \text { Bilbo Handia } & \text { Bajo Bidasoa } \\ \text { Llanada alavesa } & \text { Gernika-Bermeo } & \text { Donostialdea } \\ \text { Montaña alavesa } & \text { Markina-Ondarroa } & \text { Alto Deba } \\ \text { Rioja alavesa } & \text { Plentzia-Mungia } & \text { Goierri } \\ \text { Cantábrica alavesa } & \text { Encartaciones } & \text { Tolosaldea } \\ & \text { Ibaizabal-Nervión } & \text { Urola-Deba } \\ & \text { Duranguesado } & \text { Urola-Kostaldea }\end{array}$

Para la correcta aplicación de los objetivos se establece una Comisión de AM (art. 4) como órgano de consulta, asesoramiento y coordinación en las cuestiones relativas a la política de Agricultura de Montaña. Esta Comisión es de ámbito autonómico.

En pos de la consecución de los objetivos señalados se prevé la elaboración y ejecución de Programas Comarcales. Esta labor se podrá en- 


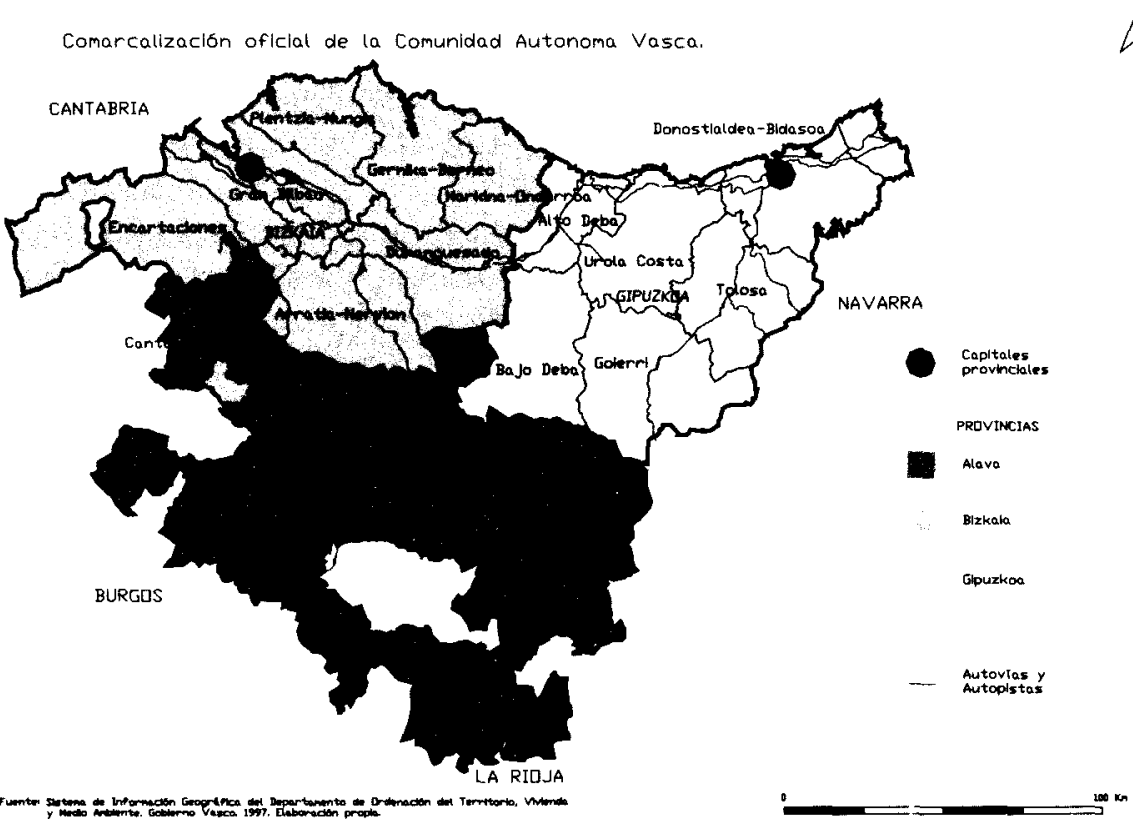

cargar tanto a un Comité Comarcal, como a entidades públicas o privadas (Asociaciones de AM). En éstos dos últimos casos, se deberá informar al Comité Comarcal.

Los Comités Comarcales de AM (art. 5) son órganos de consulta, asesoramiento y coordinación en la política de Agricultura de Montaña de la comarca y están integrados por los siguientes representantes: uno de cada municipio de la comarca, tres de la Diputación correspondiente, uno del Gobierno Vasco, uno de los Sindicatos Agrarios y un representante de las Cámaras Agrarias de la comarca.

Asimismo, se regulan las características que deben cumplir las Asociaciones de Agricultura de Montaña (art. 7):

- Serán entidades de derecho privado, con personalidad jurídica y capacidad de obrar propias, siendo su objeto servir de cauce de participación y colaboración de los agricultores, las industrias agroalimentarias, el sector agroturístico y la administración local de la comarca correspondiente en la elaboración y ejecución de los programas comarcales de Agricultura de Montaña. 
- Sólo podrán formar parte de la Asociación las entidades asociativas de los agricultores de la comarca y de las empresas de los sectores agroalimentario y agroturístico que dispongan de instalaciones y establecimientos en la propia comarca, además de Ayuntamientos, Entidades Locales menores y Parzonerías de la comarca.

- La representación de las agrupaciones de agricultores en la Asociación deberá disponer de la mayoría absoluta en los órganos de decisión de la misma, disponiendo los estatutos de la Asociación de mecanismos que impidan la pérdida de la mayoría por la representación de los agricultores.

Hasta el momento, sólo las Asociaciones de Agricultura de Montaña se han dedicado a la elaboración y ejecución de este tipo de Programas, pero el Gobierno Vasco, gracias a lo señalado en el Decreto, se guarda la opción de elección de la entidad a asignar.

Estamos, por tanto, ante una entidad en la que va a dominar una visión "agrarista" del desarrollo rural, a pesar de la amplitud de objetivos que plantea, y que de manera directa e indirecta pasa a estar dirigida por la intervención pública.

Los objetivos, por otro lado, coinciden con los habituales en los programas de desarrollo rural impulsados por la $C E$, recogidos en tres ejes principales: diversificación, en un esfuerzo por buscar actividades alternativas a una reconversión agraria inevitable; desarrollo endógeno ${ }^{6}$, puesto que se considera que sólo podrá tener éxito si éste se fundamenta en los recursos locales; integrado, capaz de garantizar un nivel de ingresos digno a través de la combinación de diferentes actividades (Corbera, 1999).

\section{ASOCIACIONES DE AGRICULTURA DE MONTAÑA EN GIPUZKOA. UN FUNCIONAMIENTO PECULIAR}

A partir de la fundación de la primera Asociación y casi a un ritmo anual, se va dotando a toda la provincia de Gipuzkoa de esta infraestructura de desarrollo rural. Las Asociaciones que actualmente funcionan en este territorio se recogen a continuación:

6 La necesidad de que sea la población local la que planifique y lidere este tipo de proyectos no sólo es recomendada por la CE sino que multitud de investigadores la consideran fundamental en el éxito de estos programas. Consultar JENKINS (2000), LeEuWIS (2000). 
COMARCA

Urola Costa

Goierri

Alto Deba

Tolosaldea

BajoDeba

Donostialdea
ASOCIACIÓN

Urkome

Goimen

Deba Garaia

Tolomendi

Debemen

Behemendi

\section{CONSTITUCIÓN}

1987

1988

1989

1990

1991

1993

\subsection{Figura legal y composición de estas Asociaciones}

Forma Jurídica: las Asociaciones de Agricultura de Montaña establecen su régimen jurídico al amparo de la Ley de Asociaciones regulada por el Gobierno Vasco. Así pues, se rigen por unos Estatutos aprobados por el Gobierno Vasco, similares a las que rigen el funcionamiento de cualquier asociación cultural. Las características más significativas son las siguientes:

- Entidades sin ánimo de lucro.

- Están integradas por los Ayuntamientos de la comarca y por las Asociaciones agrarias con representación comarcal.

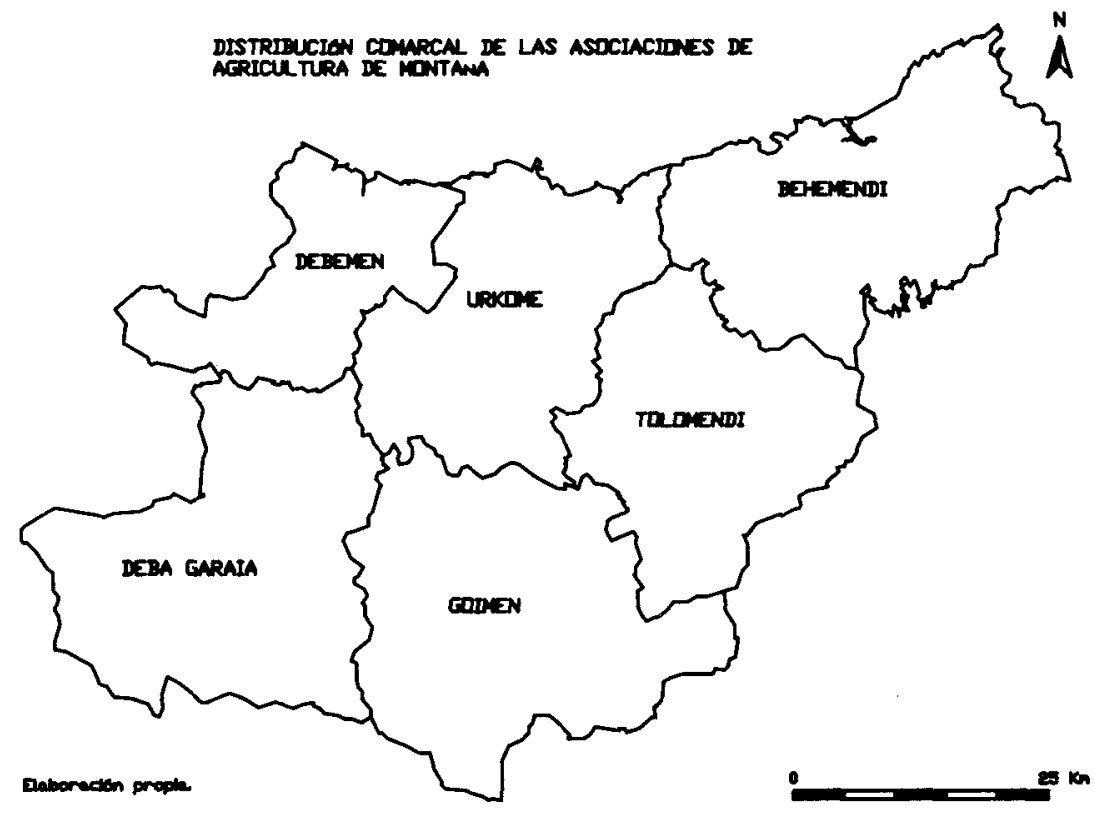


- Los cargos de las Asociaciones (presidente, tesorero, secretario, etc.) no tienen ningún tipo de remuneración, lo cual se detalla de un modo específico en los Estatutos.

Fines de la Asociación: los fines señalados en los Estatutos como propios de las Asociaciones de Agricultura de Montaña son los siguientes:

- Mejora de las condiciones de vida.

- Dotación de servicios de vida.

- Servir de cauce de participación y colaboración de los agricultores, las industrias agroalimentarias, el sector agroturístico y la Administración local de la comarca en la elaboración y ejecución de los programas hasta ahora señalados.

Órganos de decisión:

- Asamblea General. Es quien elige anualmente a la Junta Rectora, pero sólo renovando a la mitad de sus miembros.

- Junta Rectora. Cumple la función de debatir y proponer Programas comarcales. La Junta Rectora debe contar con una mayoría de integrantes de Asociaciones agrarias. Está compuesta por un número variable de miembros dependiendo de la Asociación, aunque por lo general suele rondar de los 11 a los 13 miembros.

Composición de las Asociaciones de Agricultura de Montaña. Forman parte de las Asociaciones de Agricultura de Montaña los ayuntamientos de cada comarca y las Asociaciones representativas del sector agroalimentario y turístico.

Las Asociaciones Agrarias de ámbito provincial en Gipúzkoa forman parte de todas las Asociaciones de Agricultura de Montaña, si bien puede haber otras específicas en cada comarca. Las más representativas son las siguientes:

- CÁMARA AGRARIA: anteriormente era el órgano de representación de los agricultores, hoy en día suplantado por los sindicatos.

- EHNE: sindicato agrario.

- ENBA: sindicato agrario.

- LURGINTZA: cooperativa de servicios. Asesoramiento técnico y de gestión. 
- EHNELUR: cooperativa de Servicios.

- GEBE: asociación de propietarios forestales.

- GILBE: asociación de Horticultores y Floricultores.

- FRUITEL: asociación de fruticultores.

- GIFE: asociación de ganaderos de vacuno de leche.

- ELE: asociación de oveja Latxa.

- EHBE: asociación de productores de vacuno de carne (pirenaica)

- GUE: asociación de productores de conejos.

- EBEL: asociación de mujeres del caserío.

- BIOLUR: asociación de Agricultura ecológica.

- GEE: asociación de apicultores.

- GURELESA: central lechera.

Estructura laboral. Cada Asociación de Agricultura de Montaña es libre de contratar a los trabajadores que considere oportuno. No obstante, existe una estructura parecida en las 6 Asociaciones ubicadas en Gipúzkoa. Todas ellas cuentan con un técnico, empleado a jornada completa, un administrativo, tanto a jornada completa como a media jornada, dependiendo de la Asociación, y un dinamizador a media jornada, al que compete promover y proponer proyectos, es decir, entablar contactos entre las entidades implicadas en el desarrollo rural, recogiendo ideas y proponiendo actuaciones a la Junta Rectora.

La financiación de este personal corre a cargo de los ayuntamientos de la comarca y se realiza en la mayoría de ellas teniendo en cuenta dos criterios:

- El 50\% de la cuota se calcula proporcionalmente al número de habitantes de los municipios que conforman la comarca.

- El 50\% restante se calcula proporcionalmente al número de caseríos con que cuenten los municipios integrantes de la comarca.

\subsection{Financiación de los programas elaborados por las AAMM}

La financiación de los Programas anuales de la Agricultura de Montaña se realiza con cargo a los presupuestos del Departamento de Agricultura de la Diputación Foral de Gipúzkoa. Es a partir de 1993 cuando se estable- 
cen unos criterios de distribución de los fondos entre las comarcas, así como límites de subvención. Todo ello se regula en la Norma Foral 15/1993.

Una vez asignada la partida presupuestaria por parte del Departamento de Agricultura de la Diputación, la distribución entre comarcas se realiza del siguiente modo:

- Se distribuye una cantidad fija (30 millones) para todas las Comarcas de Agricultura de Montaña.

- La cantidad restante se distribuye en base a los siguientes:

- El coeficiente comarcal de Montaña.

- El número de explotaciones agrícolas existentes en cada comarca.

- La superficie total de la comarca y la superficie agraria útil (SAU).

- Número de Unidad de Ganado Mayor (UGM).

Respecto a las subvenciones, se estableció un límite de financiación del $65 \%$ del valor de la inversión del proyecto, es decir, la suma de las diferentes líneas de ayuda establecidas por la Administración (Diputación Foral y Gobierno Vasco) no podrá superar el $65 \%$ de la inversión del total.

\subsection{Aprobación de un programa comarcal}

La aprobación de un programa anual requiere cumplir toda una serie de requisitos previos, desde la recepción de los proyectos hasta su aprobación por el Comité Comarcal. Entre otros pasos cabe señalar como más significativos los siguientes:

1) Las Asociaciones de $A M$ establecen un plazo para la presentación de proyectos que deben ser presentados por los ayuntamientos o asociaciones agrarias. No se admiten propuestas de personas individuales.

2) Tras la recepción de las proposiciones, y en base al presupuesto anual, realizan su propio programa, que es presentado al Comité Comarcal para su discusión.

3) El Comité Comarcal aprueba la ejecución del programa. Lo habitual es que éste sea aceptado tal y como ha sido presentado por la Junta de cada Asociación.

4) La ejecución práctica es realizada por aquella entidad que haya propuesto el proyecto, es decir, por ayuntamientos o asociaciones agrarias. 
La función de las Asociaciones de AM es la de control y seguimiento del programa aprobado, con la consiguiente tramitación de las ayudas.

\subsection{Actividades y posibilidades presupuestarias de las Asociaciones de Agricultura de Montaña}

Como se ha señalado, las Asociaciones de Agricultura de Montaña se encargan de la recepción, proposición y seguimiento de los programas comarcales. Estas, además, no sólo trabajan en proyectos con respaldo económico, sino que muchas de sus actuaciones se centran en actividades que no cuentan con una financiación procedente de los Programas Comarcales de Agricultura de Montaña. En otros casos, especialmente en aquellos proyectos que adquieren un carácter comarcal, son las mismas Asociaciones quienes proponen, elaboran y ejecutan las iniciativas.

A diferencia de otras líneas de ayuda, donde se exponen muy detalladamente las condiciones de acceso a las mismas, en las Asociaciones de Agricultura de Montaña no existen unos criterios fijos. Por ello, ha sido cada Asociación la que ha establecido sus criterios según el momento, proyecto, situación, etc. Se carece de un Plan de Actuación previamente elaborado que recoja los objetivos y bases para la correcta selección y elaboración de los proyectos.

Analizamos los diferentes apartados en los que se distribuyen o catalogan los proyectos, los criterios de aprobación - cuando los hay - y los niveles de financiación, tomando como referencia los utilizados en la Asociación Goimen.

Estudios: propuestas que analizan las necesidades o carencias en determinados sectores y proponer las soluciones que se consideren óptimas.

Acciones para la formación cultural: cursos de formación tanto básicos como específicos en temas determinados; viajes para conocer otros metodos de producción, la organización de otras zonas de Agricultura de Montaña, etc.

Comercialización: proyectos de transformación de producciones agrarias novedosos, siempre con la condición de que no se trate de un proyecto individual; mercados especiales y semanales. Propuestas de actuaciones que tienen una influencia directa en los agricultores; publicidad 0 promoción de dichos mercados u otros productos.

Reestructuración: proyectos para la reconversión de la producción de los caseríos (potenciación de nuevas producciones); iniciativas que apro- 
vechen los recursos propios de los caseríos o las actividades que ofrecen éstos.

Conservación de los suelos. Paisaje: actuaciones que no siendo rentables para el agricultor, son necesarios de cara a la sociedad y al futuro (plantación de árboles autóctonos, drenajes de praderas y laderas con problemas de deslizamientos etc.).

Equipamientos y servicios: transporte para la escolarización; mejora de las condiciones de vida de los pastores transumantes; renovación de los locales sociales de los barrios rurales; dotación de equipamientos en general a barrios rurales: luz, frontones, etc.; otros servicios: instalación de buzones rurales, paneles de información, etc.; maquinaria de servicio: proyectos que tienden a racionalizar las inversiones, proponiendo la compra en común, dirigido a municipios y asociaciones (desbrozadoras para la limpieza de los caminos, palas para la limpieza de la nieve, hormigoneras, ...).

Actividades complementarias a la producción agraria: iniciativas que propongan otra fuente de ingresos además de la procedente de la Agricultura (proyectos relacionados con trabajos de tipo artesanal, proyectos que impulsen el aprovechamiento de otros recursos propios del caserío; visitas de escolares a las explotaciones agrarias).

Recuperación del entorno: iniciativas que procuren la recuperación y adecentamiento de espacios naturales o construcciones típicas (acondicionamiento de fuentes, arreglo de bordas, mejora de los parques, conservación de las ermitas, etc.).

Infraestructuras: abastecimiento de agua. Condiciones necesarias para la aprobación de los proyectos: vivir en el caserío / tener una actividad agraria / agua destinada a consumo humano; caminos rurales. Condiciones para la aceptación de accesos a caseríos: vivir en el caserío / tener una actividad agraria / primer acceso al caserío; muros contención (escollera).

Como se observa, las Asociaciones cuentan con un presupuesto abierto a multitud de acciones si bien todas tienen un elemento común, el sujeto, en todos los casos coincidente con el agricultor, con el medio que directamente trabaja o con sus necesidades. Por otro lado, si tenemos en cuenta que el orden en que hemos recogido esta tipología de proyectos marcaría los criterios de preferencia a la hora de recibir las subvenciones, observamos cómo aquellas iniciativas ligadas directamente a la producción o comercialización agrícola adquieren preferencia. En un segundo lugar se situarían las dirigidos a complementar en el caserío los rendimientos obtenidos de la actividad agrícola y, finalmente, las infraestructuras de acceso a la vivienda, agua y caminos. 


\section{UNA PRIMERA FASE ORIENTADA A LA CONSECUCIÓN DE UNA INFRAESTRUCTURA ADECUADA. PERIODO 1992-1998}

A pesar de que el programa de Agricultura de Montaña no ha presentado variaciones de relevancia en el territorio guipuzcoano desde que se inició, optamos por diferenciar dos etapas distintas desde que el programa comienza a funcionar en todo Gipuzkoa, relacionadas con los objetivos políticos que en cada uno de ellos se plantean y que se reflejan en la tipología de proyectos que se materializan.

Para poder disponer de una idea más concreta del destino que tienen los fondos y en qué cuantía, vamos a recoger de manera resumida los conceptos y partidas presupuestarias aprobadas por parte de la Asociación que mayor diversidad de actuaciones ha desarrollado en el periodo 1993-1998, la correspondiente a la comarca de Goierri.

Tabla 1. Proyectos aprobados por goimen entre 1993 y 1998

\begin{tabular}{lrrr}
\hline & N. & $\%$ & $\begin{array}{c}\text { Subvención } \\
(P t s .)\end{array}$ \\
\hline ESTUDIOS & 3 & & 1.992 .000 \\
Incendios: Estudio de planificación (94) & 1 & 65 & 1.000 .000 \\
Iniciativa para la evolución de Telleriarte (95) & 1 & 65 & 836.000 \\
La situación social de las mujeres baserritarras (96) & 1 & 65 & 156.000 \\
ACTUACIONES PARA LA FORMACIÓN CULTURAL & 8 & & 5.292 .353 \\
Viajes de las Asociaciones de Agricultura de Montaña & 4 & - & 4.032 .353 \\
Publicación de libros & 4 & & 1.260 .000 \\
ACTUACIONES COMERCIALES & 28 & & 18.414 .489 \\
Mercados & 14 & - & 6.790 .500 \\
El nuevo centro de «Artzai Gazta» (94) & 1 & 35 & 2.424 .546 \\
Centro de transformación de Zerain & 2 & - & 2.158 .000 \\
Matadero de pollos «Lumagorri» (94-95) & 2 & - & 3.457 .285 \\
"Baserriko produktuak» & 7 & - & 1.534 .158 \\
Centro de miel de Gipuzkoa (98) & 1 & - & 1.800 .000 \\
Estudio de centros de transformación & 1 & - & 250.000 \\
MANTENIMIENTO DE TIERRAS & 3 & & 1.946 .380 \\
Trabajos de drenaje & 3 & & 1.946 .380 \\
SERVICIOS Y EQUIPAMIENTO & 68 & & 127.957 .000 \\
Casetas de buzones & 6 & - & 1.104 .000 \\
Transporte escolar (5 pedidos) & 25 & 60 & 7.589 .000 \\
Repetidores de TV & 1 & - & 307.000 \\
Paneles de información, placas de caserio... & 10 & 60 & 1.972 .000 \\
Locales sociales & 8 & - & 9.650 .000 \\
Equipamientos para obras de barrio (frontón, iglesia...) & 1 & - & 329.000 \\
Mejoras en Aralar y Aizkorri & 12 & 50 & 96.621 .000 \\
Aserradero ZERRALAN (96) & 1 & - & 6.900 .000 \\
& & &
\end{tabular}


Tabla 1. Proyectos aprobados por goimen entre 1993 y 1998 (continuación)

\begin{tabular}{lrrr}
\hline & $N{ }^{\circ}$ & $\%$ & $\begin{array}{r}\text { Subvención } \\
(\text { Pts.) }\end{array}$ \\
\hline Servicio de esparzidora de purin (96-98) & 2 & - & 2.040 .000 \\
Servicio de recogida de oferta de tierras (98) & 1 & - & 195.000 \\
Limpieza de caminos rurales (98) & 1 & 25 & 1.250 .000 \\
MAQUINARIA AGRícoLA & 13 & & 4.011 .790 \\
Maquinaria para realización de servicios & 13 & - & 4.011 .790 \\
ACTIVIDADES COMPLEMENTARIAS & 19 & & 24.796 .350 \\
Visita a Caseríos & 12 & & 9.290 .350 \\
El granero de Zerain (93) & 1 & & 13.505 .000 \\
LENBUR & 3 & 35 & 1.041 .000 \\
Diseño de paquetes turisticos & 3 & - & 960.000 \\
ESPACIOS NATURALES & 12 & & 2.534 .929 \\
Reparación de fuentes & 1 & - & 110.000 \\
Reparación de casetas & 11 & $55-65$ & 2.424 .929 \\
INFRAESTRUCTURAS & 159 & & 186.408 .151 \\
Suministro de agua & 70 & & 74.706 .040 \\
Caminos rurales & 88 & - & 110.285 .111 \\
Saneamientos & 1 & - & 1.417 .000 \\
\hline \multicolumn{1}{c}{ TOTAL } & 313 & & 373.353 .442 \\
\hline
\end{tabular}

Fuente: Asociación de Agricultura de Montaña Goimen, 1999.

Las inversiones efectuadas, agrupadas atendiendo a su tipología, nos ofrecen los siguientes resultados:

Tabla 2. Inversiones aprobadas por goimen entre 1992-98

\begin{tabular}{|c|c|c|c|}
\hline & N. ${ }^{\circ}$ Proyectos & Sub. Directa (pts.) & $\%$ \\
\hline Estudios & 3 & 1.992 .000 & $0,5 \%$ \\
\hline Formación & 7 & 4.292 .353 & $1,1 \%$ \\
\hline Comercialización & 28 & 18.414 .489 & $4,9 \%$ \\
\hline Reconversión & 0 & 0 & $0 \%$ \\
\hline Conservación suelo & 3 & 1.946 .380 & $0,5 \%$ \\
\hline Servicios - equipam. & 68 & 127.957 .000 & $34,3 \%$ \\
\hline Maquinaria & 13 & 4.011 .790 & 1,1 \\
\hline Act. complementarias & 20 & 25.796 .350 & $6,9 \%$ \\
\hline Recuperación entorno & 12 & 2.534 .929 & $0,7 \%$ \\
\hline Infraestructuras & 159 & 186.408 .151 & $50 \%$ \\
\hline TOTAL & 313 & 373.353 .442 & $100 \%$ \\
\hline
\end{tabular}

Fuente: Asociación de Agricultura de Montaña Goimen, 1999. 
De todas las tipologías analizadas es la realización de infraestructuras, agua y preferentemente caminos, la que acapara alrededor de la mitad de las inversiones efectuadas en este periodo. La necesidad de dotar a todo caserío habitado de un acceso pavimentado y una toma de agua potable, es una condición previa a cualquier programa de desarrollo de actividades en el medio rural que se plantee y ello se refleja en los datos que aportamos?.

El alto porcentaje de gasto en servicios y equipamientos de la zona rural es otro de los aspectos a destacar, algo más de un tercio de las inversiones efectuadas, una situación relacionada con la falta de equipamientos básicos por parte de la población que habita el caserío. En este concepto se incluye el transporte escolar, acondicionamiento de locales sociales así como determinados servicios relacionados con la actividad agraria (purín de ganado). Sin embargo, algo más del $70 \%$ de la inversión se concentra en una única partida, coincidente con las mejoras efectuadas en los comunales de Aralar y Aizkorri, parzonerías que cuentan con las mayores superficies de pastos de Montaña en la provincia de Gipuzkoa ${ }^{8}$. Las ayudas han sido destinadas preferentemente al acondicionamiento de bordas o majadas en las que los pastores pasan la temporada estival (acceso, electricidad, agua, habitación etc.). En definitiva, observamos cómo alrededor del $80 \%$ del presupuesto es dirigido a la realización de infraestructuras viarias y al abastecimiento de agua.

Otra serie de iniciativas, como puede ser la promoción de mercados, la organización de visitas de escolares a caseríos e incluso la consecución de albergues, como el caso de Zerain, también han sido impulsadas. Sin

\footnotetext{
La apuesta por parte de la entidad foral en la realización de infraestructuras es anterior al programa de agricultura de montaña. Este no hace sino recoger algunos de los trabajos que la Diputación realizaba antes de su creación, como podemos observar en la siguiente cita. "La solución con hormigón se viene imponiendo progresivamente a las restantes alternativas, de forma que en el año 1988 ya no se construye ningún camino con triple riego habiendo bajado la pavimentación con aglomerado asfáltico de $25 \mathrm{~km}$. en 1982 a $4 \mathrm{~km}$. en 1989 . Una de las razones de este predominio del hormigón es su sencillez de construcción puesto que en la ejecución se utilizaba un procedimiento de trabajo en común existente en el mundo rural vasco: el auzolan, que en euskera significa trabajo no retribuido que se hace para cubrir las necesidades que afectan a la comunidad vecinal, tales como la mejora de los caminos, abastecimientos de aguas, o la reparación de ermitas o caseríos que han sufrido un incendio" (LOSA y GARCía, 1991, pág. 3).

8 Estas parconerías se corresponden habitualmente con la definición de monte. Según señala URZAINOUI "si bien el término monte en su aceptación legal responde a una valoración productivo-forestal que deriva básicamente de sus características ambientales, hay que decir que en el caso concreto de Gipuzkoa, la expresión de montes comunales y/o montes de utilidad pública alcanza un significado también topográfico ya que se trata de tierras altas y accidentadas, de formas con pendientes acusadas y abruptas y, por consiguiente, de escasa o nula aptitud agricola, de recursos agrarios limitados y de clara vocación forestal y ganadera" (1987, pág. 181)
} 
embargo, la cuantía de todas éstas es sensiblemente más reducida e incluso recogida en actuaciones muy concretas y puntuales.

Los programas impulsados en el periodo analizado por la Asociación de Agricultura de Montaña Goimen se adecúan a los objetivos inicialmente propuestos pero se concentran casi exclusivamente en la dotación de infraestructuras a este medio rural. El resto de objetivos, especialmente aquellos destinados a constituir un grupo de actividades capaces de complementar la renta de los agricultores, quedan relegados a un segundo plano siendo su presencia prácticamente testimonial.

Como hemos señalado, Goimen se nos presenta en este periodo como una de las Asociaciones más dinámicas y que mayor diversidad aparente de proyectos presenta. En el resto de Asociaciones de Gipuzkoa, la importancia que adquieren las inversiones en infraestructuras es todavía mayor.

Tabla 3. Inversiones aprobadas en los programas de agricultura de montaña de Gipuzkoa entre 1992 y 1998

\begin{tabular}{lrrrrrrr}
\hline & Otras activid. & $\%$ & Servi-equip & $\%$ & Infraestruc. & $\%$ & Presupuesto \\
\hline Urkome & 48.934 .713 & $14 \%$ & 59.574 .748 & $17 \%$ & 242.385 .212 & $69 \%$ & 350.894 .673 \\
Goimen & 34.949 .843 & $9 \%$ & 149.089 .749 & $40 \%$ & 186.409 .448 & $50 \%$ & 370.449 .040 \\
Deba Garai & 53.201 .184 & $18 \%$ & 14.517 .807 & $5 \%$ & 234.336 .918 & $78 \%$ & 302.055 .909 \\
Tolosa & 109.632 .984 & $32 \%$ & 25.117 .690 & $7 \%$ & 207.621 .984 & $61 \%$ & 342.372 .658 \\
Debemen & 13.925 .624 & $5 \%$ & 71.893 .671 & $27 \%$ & 182.927 .757 & $68 \%$ & 268.747 .052 \\
Behemendi & 49.868 .987 & $14 \%$ & 52.986 .499 & $15 \%$ & 248.703 .109 & $71 \%$ & 351.558 .595 \\
\hline \multicolumn{1}{c}{ TOTAL } & 310.513 .335 & $16 \%$ & 373.180 .164 & $19 \%$ & 1.302 .384 .428 & $66 \%$ & 1.986 .077 .927 \\
\hline
\end{tabular}

Fuente: Asociaciones de Montaña de Gipuzkoa, 1999.

La realización de infraestructuras, si cabe, adquiere aún más relevancia en el resto de comarcas de Gipuzkoa. En la mayoría de ellas también se ponen en marcha distintas iniciativas destinadas a la mejora de la calidad de vida de la población que habita en el caserío, entre las que destacan el correo rural, el transporte escolar o la adecuación de locales sociales e incluso deportivos pero, en todos los casos, se trata de actuaciones puntuales o que requieren pequeñas inversiones.

El análisis de estos programas deja entrever que la necesidad prioritaria del desarrollo rural en Gipuzkoa pasa estos años por la consecución de una estructura viaria y de abastecimiento de agua suficiente. El resto de necesidades e iniciativas, que también atañen al desarrollo rural y que se recogen en el decreto de Agricultura de Montaña, son desatendidas. 
La consecución de unos programas de desarrollo rural destinados en exclusividad a la realización de infraestructuras, cuando su estructura de funcionamiento, con la participación mayoritaria de Asociaciones y Sindicatos agrarios, hace prever el respaldo a otro tipo de inciativas, resulta cuanto menos paradójica e indica que estas entidades, al menos en este periodo, no han funcionado como inicialmente se preveía. En este sentido, observamos que la importante y necesaria presencia de los ayuntamientos, que asumen los costos de financiación del personal empleado, no se constituye en un motor para el desarrollo endógeno de este medio sino que, fieles a su qué hacer, impulsan la realización de aquellos proyectos que a ellos les competen, básicamente infraestructuras. Frente a los grupos locales o profesionales, carentes a menudo de personal, tiempo e iniciativa, los ayuntamientos cuentan con medios técnicos suficientes como para realizar las correspondientes peticiones. Difícilmente, el personal técnico de la Asociación, dependiente de las aportaciones municipales, se opondrá a este tipo de actuaciones.

Sin poner en tela de juicio la conveniencia de las inversiones en infraestructuras que se realizan estos años, llegamos a la conclusión de que se abusa del recurso a ellas en unos programas que, en principio y en orden de prioridad, tienen otros deberes que cumplir ${ }^{9}$.

\section{LOS PROYECTOS SECTORIALES COMO REFERENTE. AÑOS 1999-2001}

La etapa que se inicia en 1998 y que comienza a reflejarse en los presupuestos de 1999, viene ligada a un cambio en la Dirección de Agricultura de la Diputación Foral, que tiene su traslado en la Política de Agricultura de Montaña que hasta esa fecha se había puesto en marcha. La nueva dirección, a instancia de los sindicatos agrarios, apuesta por dirigir estos programas a la consecución de proyectos de desarrollo agrícola, limitando el porcentaje máximo que cada Asociación puede destinar a la realización de infraestructuras.

Los programas que estos años se aprueban son un reflejo de la pérdida de influencia municipal y del mayor papel que adquieren ahora las aso-

9 El tipo de iniciativas impulsado, a pesar de que atendiendo al decreto que los regula presentan unos objetivos similares a los programas Leader, son totalmente distintos. Frente al domino de las infraestructuras en nuestro caso, en los programas Leader es el turismo rural e incluso en algún caso la pequeña empresa la que acapara las mayores inversiones. Consultar OBIOL, 1998, Badas et al. 1998, LarRull, 1998, Corbera, 1999). 
ciaciones y sindicatos agrarios. De entre todas, tal vez sean las de las comarcas de Donostialdea-Bidasoa y de Tolosaldea las que mejor reflejen este cambio.

La Asociación de Tolosaldea, Tolomendi, presenta una evolución significativa en los tres años que recogemos en este periodo. En 1999 destina a la realización de infraestructuras alrededor del $56 \%$ de su presupuesto y al acondicionamiento de bordas en los pastos comunales otro $10 \%$, siguiendo los mismos criterios que los años anteriores. La única novedad estriba en el considerable importe que se destina a la manipulación y promoción de la alubia de Tolosa, si bien también en este caso se trata de una actuación puntual.

En el año 2000 comienzan a intuirse algunos de los cambios que van a caracterizar a estas asociaciones. Las infraestructuras continúan siendo la partida que mayor presupuesto acapara, el $45 \%$, aunque esta primacía es compartida con la dedicada a servicios agrícolas (recogida de plástico, ensilado de hierba, servicio de esparcimiento de purín, recogida de ganado muerto etc.) o a la consecución de estructuras productivas y de comercialización. Al siguiente año ya no se realizan infraestructuras básicas y son los servicios agroganaderos y la consolidación de estructuras productivas y de comercialización las que acaparan el $90 \%$ del presupuesto destinado a esta Asociación.

La distribución de las partidas presupuestarias que gestiona la Asociación de Agricultura de Montaña de Donostialdea-Bidasoa, Behemendi, presenta un desarrollo similar ${ }^{10}$. Si en 1999 destina a infraestructuras el $65 \%$ de su presupuesto para inversiones, en el 2000 es ya el $24 \%$ y al siguiente año desaparece este concepto. Frente a él, la realización de infraestructuras productivas, preferentemente polígonos hortícolas, la puesta en marcha de servicios generales, como pueden ser sistemas especiales de recogida de basura domiciliaria, la potenciación de actuaciones tendentes a recuperar suelo para usos ganaderos y, de manera más significativa, la puesta en marcha de servicios destinados a la actividad primaria, constituyen la tipología de proyectos que son impulsados ahora en esta Asociación.

10 Como se observa, ambas Asociaciones participan de una estrategia común, con un desarrollo rural, en este caso preferentemente agrícola, y unas iniciativas similares, diseñadas paralelamente. Esta es una de las ventajas, para algunos la principal, que ofrecen los programas de desarrollo rural y su concreción en entidades de desarrollo, las sinergias que se producen entre zonas diversas, con una tipología de proyectos que se van repitiendo de una a otra. Consultar VAN DeR Ploeg et. al. (2000), BRISTOW (2000), Esparcia et. al. (2000). 
Tabla 4. Inversiones aprobadas por Behemendi en 2001

\begin{tabular}{lrr}
\hline Proyectos & Inversión & Subvención \\
\hline Agroaldeas de Irun y Lezo & 25000000 & 15700000 \\
Servicios & 40000000 & 27100000 \\
Recuperación de suelo & 15500000 & 10075000 \\
Comercialización y Producción & 45500000 & 3000000 \\
Otros & 115000 & 115000 \\
\hline \multicolumn{1}{c}{ TOTAL (pts.) } & $\mathbf{1 2 6 1 1 5 0 0 0}$ & $\mathbf{5 5 9 9 0 0 0 0}$ \\
\hline
\end{tabular}

Fuente: Asociaciones de Montaña de Donostialdea-Bidasoa, 2001

Como se observa, aunque se apoyan distintas iniciativas de desarrollo rural, son aquellas dirigidas a dinamizar las actividades agroganaderas las impulsadas. Las Asociaciones asumen el papel de promotor del espacio y de la población agrícola de su comarca. En esta etapa se está pasando de un objetivo inicial amplio, que buscaba el desarrollo general del medio rural de cada comarca, a la estructuración y potenciación de su producción primaria.

El cambio en la tipología de proyectos que son ahora subvencionados acarrea, además de la labor de dinamización, la asunción por parte de las Asociaciones de Agricultura de Montaña de unas funciones que hasta el momento ni se habían planteado, como es la planificación, gestión y aportación de todo tipo de servicios dirigidos a la población rural. En apenas tres años, crean, dirigen y gestionan servicios agroganaderos (ensilado, siega, abono de praderas, etc.), destinados a toda la población rural (recogida de basuras, desbroces de caminos, limpieza de cunetas, etc.) e incluso medioambientales (recogida de plástico, ganado muerto, control de vertidos ilegales ${ }^{11}$, etc.).

Las Asociaciones, ante la falta de organizaciones agrícolas capaces de satisfacer las necesidades sectoriales, asumen estas funciones, hasta el punto de llevar camino de convertirse en el centro de servicios del medio rural de cada comarca.

A pesar de que algunas Asociaciones gestionan otros fondos de desarrollo rural distintos (LEADER+), lo que les permite diversificar y promover actuaciones más acordes con el concepto de desarrollo rural (turismo, pe-

\footnotetext{
11 Obsérvese que algunos de los servicios que aporta esta asociación no están directamente subvencionados por sus propios fondos sino que se trata de funciones que han ido progresivamente asumiendo con una financiación totalmente independiente. En el caso de los vertidos, la asociación cuenta con una brigada de control y recogida financiada por la mancomunidad de la Comarca de Donostia-San Sebastián.
} 
queña empresa, artesanía, etc.), la escasa implicación que tienen sobre el territorio guipuzcoano, la composición de las Juntas de estas Asociaciones e incluso la propia formación de su personal técnico está llevando a que sean los proyectos de desarrollo agrícola los prioritarios y, ante la falta de iniciativa en el sector, a que asuma la propia asociación el compromiso de liderazgo de los mismos.

\section{UNA DÉCADA SIN DEFINICIÓN DEL PROGRAMA DE AGRICULTURA DE MONTAÑA. A MODO DE CONCLUSIÓN}

Las Asociaciones de Agricultura de Montaña de Gipuzkoa, a pesar de llevar más de una década trabajando en desarrollo rural, no han servido para frenar el progresivo abandono de la actividad agraria y del espacio rural. El medio rural vasco participa de la problemática que nos encontramos en todo el estado ${ }^{12}$ y en muchas áreas de Europa: población envejecida, proceso de extensificación, falta de relevo generacional, etc.

En el caso guipuzcoano, el proceso hacia la desaparición de la explotación agraria, del caserío, avanza con gran rapidez. En este momento se está produciendo un paso hacia la extensificación. Las explotaciones abandonan progresivamente la que ha sido hasta ese momento la actividad principal, el bovino de leche, sustituyéndola por bovino de carne, actividad que está obteniendo unos resultados económicos medios por explotación muy inferiores. Esta evolución se produce, sobre todo, en aquellas explotaciones con población titular envejecida o que la ejerce a tiempo parcial, como un paso más hacia el abandono de la actividad. El proceso es tan intenso que se calcula que tan sólo un $10 \%$ de las existentes actualmente mantendrán una actividad mínima en un período de 10-15 años.

El abandono agrario, sin embargo, no conlleva la desaparición del caserío ni la pérdida de población rural que, en función de los últimos datos del padrón, se presenta como una de las más dinámicas del País Vasco ${ }^{13}$.

12 Emigración, envejecimiento y masculinidad constituyen las características más significativas de la estructura de la población del medio rural del País Vasco, partícipe, por otro lado, de un comportamiento general en todo el agro español CAMARERo marca para toda España una dinámica y una estructura similar, caracterizada por una fuerte emigración en los años sesenta y especialmente en los setenta, con un cambio de dinámica a partir de la década de los ochenta, presentando el medio rural un crecimiento vegetativo negativo, siendo los jóvenes los que emigran y especialmente las jóvenes (1997, págs. 233-237)

13 La evolución de la población de los municipios rurales vascos entre 1991 y 1996 indica que la inclinación apuntada en la década de los ochenta comienza a materializarse y estas villas ya no 
Sin embargo, la retirada de las funciones agrarias supone perder el elemento más característico de este medio y de su población, en definitiva, aceptar su absorción por parte del elemento humano ${ }^{14}$.

Las Asociaciones de Agricultura no han podido hacer frente a aquellas características propias de los espacios rurales y que limitan la acción de las distintas iniciativas de desarrollo rural, como es la despoblación o, en nuestro caso, una estructura demográfica envejecida y una falta de liderazgo notable. A pesar de que los objetivos están definidos y que el programa es abierto y permite incorporar distintos tipos de proyectos, se ha de reconocer que se carece de una visión de desarrollo integrado. De hecho, no han realizado un debate previo sobre la política rural que quieren liderar. Como hemos observado, son los criterios políticos del momento los que definen su actuación, con giros importantes dependiendo del equipo que esté al frente de la dirección de desarrollo rural encargada de la gestión del programa de Agricultura de Montaña, la Diputación Foral.

Otra de las deficiencias que constatamos la localizamos en el funcionamiento de la Asociación. Todas ellas cuentan con una Junta Directiva, compuesta mayormente por entidades agrarias, aunque estén abiertas a la incorporación de otras tipologías de socios. Sin embargo, la aportación que sus integrantes pueden hacer es muy reducida puesto que las directrices a aplicar vienen marcadas desde las instituciones públicas. Incluso la labor de dinamización de los técnicos de la Asociación queda relegada a un segundo plano ante la necesidad de priorizar los objetivos políticos planteados.

Asimismo, se presentan como unas entidades débiles, dependientes de un poder público que les otorga un presupuesto reducido y que fácilmiente pudiera prescindir de ellas. No están suficientemente asentadas en

pierden residentes, presentando respecto a 1991 un incremento del número de habitantes (5\%), muy importante en Alava y algo más atenuado en Bizkaia y Gipuzkoa. Las últimas tendencias de la población rural, según el padrón de 1996, parecen denotar una recuperación y un crecimiento positivo de la población. García SANz, analizando la población rural de España, indica cómo los pueblos rurales vascos, por debajo de 2000 habitantes, presentan un crecimiento positivo, motivado por haber sido capaces de atraer población. Sin embargo, no sólo el crecimiento del medio rural vasco sino de todo el español no depende ya del crecimiento vegetativo sino de la emigración, consecuencia de un proceso de desconcentración de las zonas más activas (1997, págs. 293-299). En el caso de Euskal Herria, GaRAYO apunta una ralentización del ritmo de decrecimiento de la población ya a partir de la década de los ochenta (1998, pág. 16).

14 "Rendirnos a la evidencia de la crisis ganadera de los espacios marginales, considerarla irreversible, supone admitir la agonia inevitable de una parte del mundo rural, precisamente de aquellas que han conservado mejor sus esencias culturales. Su total transformación en un mero espacio de ocio no supone su revitalización en condiciones distintas, superadoras del antagonismo entre el medio rural y urbano; significa su absorción» (CORBERA, 1999, pág. 235). 
el sector, ni cuentan con recursos económicos propios, con lo que, ante la retirada de la financiación pública, estas entidades desaparecerán ${ }^{15}$.

A pesar de no haberse cumplido las expectativas suscitadas, las Asociaciones han ido cumpliendo objetivos anualmente impulsando actuaciones que han tenido sus implicaciones en el medio rural de cada comarca. Entre otras resaltamos las siguientes:

1) Mejoras en el equipamiento social e infraestructuras: a este nivel, han realizado un excelente trabajo, con la implicación de los entes locales en su financiación, de tal manera que prácticamente todas las explotaciones agrarias cuentan con un camino pavimentado y con agua potable. La inversión realizada ha sido importante, destinando a esta labor dos terceras partes del presupuesto con el que han contado.

2) Acciones en servicios al medio rural: estas Asociaciones están poniendo en marcha multitud de servicios dirigidos a la función agrícola y a la zona rural. Maquinaria agrícola con intervención municipal (ensilado de hierba), recogida de ganado muerto en explotaciones agrarias, servicio de sustitución en caso de baja por accidente o enfermedad o por periodo vacacional, servicio de recogida de basura, servicio de recogida y esparcimiento de purín de ganado, etc.

3) Mejoras en la comercialización: el agro guipuzcoano, fundamentado en la pequeña explotación y en la proximidad al mercado, tiene en la venta directa una de sus fuentes de ingreso principales. En este campo se han efectuado un número importante de acciones, que van desde la consolidación de los mercados tradicionales a la creación de nuevas modalidades de mercado e incluso a su incorporación a nuevos circuitos de venta. La venta al por mayor también ha sido potenciada, con la consecución en los mercados principales de abasto de la capital de varias naves dedicadas a la venta de productos locales.

Aún así, todas estas medidas no han sido suficientes y el sector agrario decrece constantemente, a un ritmo vertiginoso. El medio rural, el caserío en Gipuzkoa no se deshabita pero pierde el carácter agrícola que le ha caracterizado.

15 Una situación, por paradójica que parezca, común a las entidades de desarrollo rural surgidas al amparo de los programas impulsados por la CE. Su creación a partir de un programa de desarrollo rural, su dependencia del dinero público y su control por parte de la administración son características que, atendiendo a las afirmaciones de EsPARCIA et. al. (2000), se generalizan en la mayoría de ellos. 
El futuro de estas Asociaciones pasa por redefinir claramente sus funciones y objetivos, que se han de recoger en la elaboración de programas que presenten una estabilidad temporal. En este momento combinan en muchos casos su progresiva reconversión en una cooperativa de servicios, preferentemente agrícola, con la responsabilidad que le otorga la ejecución del programa LEADER +. Un sin fin de funciones que ni permiten marcar unas líneas de actuación ni dar respuesta a un medio con opciones también en otros sectores productivos.

\section{BIBLIOGRAFÍA}

AINz lBarRondo, M. J. (1999): El caserio vasco: territorio para un país neoindustrial. Universidad del País Vasco. Tesis Doctoral inédita. Vitoria-Gasteiz.

badás Fernández, J., De Uña Álvarez, E. y GonzÁlez Caiña, J. (1998): “Aplicación de las políticas comunitarias: dos ejemplos en el medio rural del S.E. Gallego". IX Coloquio de Geografia Rural. Universidad del País Vasco. Vitoria-Gasteiz, págs. 429-439.

BRistow, G. (2000): "Structure, strategy and space. Issues of progressing integrated rural development in Wales" European urban and regional students. n. ${ }^{\circ}$, págs. 19-33.

CORBERA MILLAN, M. (1999): “Las políticas de desarrollo rural en la región Cantábrica: los programas LEADER». Cambios en los Espacios Rurales Cantábricos tras la integración de España en la UE. Universidad de Cantabria, págs. 175-237.

ESPARCIA, J., MOSELEY, M. y NOGUERA, J. (2000): Exploring rural development partnerships in Europe. Informes Uderval. Universidad de Valencia.

ESPARCIA, J., y NOGUERA, J. (1998); "La eficacia de las políticas de desarrollo rural». IX Coloquio de Geografia Rural. Universidad del País Vasco. Vitoria-Gasteiz, págs. 77-84.

Garayo, J. M. (1998): “Población y sociedades rurales" en MEAZA, G. y RUIZ, E. (dirs.): Geografia de Euskal Herria. vol. VI (Los espacios y actividades rurales e industriales). Etor, Donostia, págs. 9-23.

Gobierno VASCO. Departamento de Agricultura (1996): Iniciativas Comunitarias Leader II. Vitoria-Gasteiz.

JENKINS, T. N. (2000): «Putting postmodernity into practice: endogenous develpment and tehe role of traditional cultures en the rural development of marginal regions" Ecological Economics, n. ${ }^{\circ} 34$, págs. $301-314$.

LARRUL ChimisanAS, A. (1998): «Desarrollo rural y la diversidad productiva: el caso del Leader I en la tierra alta". IX Coloquio de Geografía Rural. Universidad del País Vasco. Vitoria-Gasteiz, págs. $429-439$

LEEWIS, C. (2000): «Reconceptualizing participation for sustainable rural development:Towards a negotiation approach» Development and Change. n. ${ }^{\circ} 31$, págs. 931-959.

Obiol Menero, E. (1998): "Turismo rural e iniciativa Leader. La experiencia del Pais Valenciano". IX Coloquio de Geografia Rural. Universidad del País Vasco. Vitoria-Gasteiz, págs. 429-439.

Uazainki Miqueleiz, M. A. (1990): "Comunidades de Montes en Guipúzcoa: Las Parzonerias". Mundaiz. San Sebastián.

Van Der Ploeg, J. D., Renting, H. (2000): «Impact and potential: A comparative review of European rural development practices" Sociologia ruralis, n. ${ }^{\circ} 40$, págs. 529-543. 